\title{
Efficient Iterative Methods for FIR Least Squares Identification
}

MURALI TUMMALA

Abstract-Two iterative methods based on matrix splitting are presented so as to facilitate the least squares identification of finite impulse response (FIR) systems. The first method yields an order recursive solution to a recently proposed fixed order iterative procedure. The second is a simplified and computationally efficient method and is applicable when the input signals are white, or nonwhite with a correlation coefficient of value less than $1 / 3$. Convergence performance of both algorithms is briefly discussed.

\section{INTRODUCTION}

The problem of solving least squares identification of finite impulse response (FIR) or moving average (MA) systems based on an iterative method by matrix splitting has recently been proposed by Ohsmann [1]. The iterative procedure involves the inversion of a Toeplitz matrix for a given fixed order case. As mentioned by Ohsmann, his algorithm requires only a few iterations to obtain satisfactory results and uses $\mathcal{O}\left(N^{2}\right)$ arithmetic operations per iteration. In this paper we wish to extend Ohsmann's approach to obtain an order recursive solution by using the linear prediction lattice algorithm treated by Marple [2]. An attractive feature of the proposed order recursive algorithm is that it provides the Toeplitz matrix inversion as Cholesky decomposition at no extra computational requirement. Additionally, we discussed a simplified matrix splitting iterative method which provides an efficient solution for situations when the input process is nearly white or has a fast decaying autocorrelation function.

In an FIR system identification model, error between a reference (unknown) system output $y(k)$, and the FIR filter output $\hat{y}(k)$, is given by

$$
e(k)=y(k)-\hat{y}(k)=y(k)-\sum_{m=0}^{M} a_{m}^{M} x(k-m)
$$

where $a_{m}^{M}$ are the $M$ th order FIR filter impulse response values and $x(k)$ is the input data. Now (1) can be represented in the vector form as follows:

$$
\boldsymbol{e}(k)=\boldsymbol{y}(k)-X_{M} \boldsymbol{a}_{M}
$$

where

$$
\begin{aligned}
& \boldsymbol{y}^{T}(k)=[y(k-N+M) y(k-N+M+1) \cdots y(k-1) y(k)] \\
& \boldsymbol{a}_{M}^{T}(k)=\left[\begin{array}{llll}
a_{0}^{M} & a_{1}^{M} & \cdots & a_{M-1}^{M} a_{M}^{M}
\end{array}\right] \\
& X_{M}=\left[\begin{array}{cccc}
x(k-N+M) & x(k-N+M-1) & \cdots & x(k-N) \\
x(k-N+M+1) & x(k-N+M) & \cdots & x(k-N+1) \\
x(k-N+M+2) & x(k-N+M+1) & \cdots & x(k-N+2) \\
\vdots & \vdots & & \vdots \\
x(k-2) & x(k-3) & \cdots & x(k-M-2) \\
x(k-1) & x(k-2) & \cdots & x(k-M-1) \\
x(k) & x(k-1) & \cdots & x(k-M)
\end{array}\right] .
\end{aligned}
$$

Manuscript received April 2, 1988; revised June 28, 1989. This work was supported by the Naval Postgraduate School Research Council.

The author is with the Department of Electrical and Computer Engineering, Naval Postgraduate School, Monterey, CA 93943

IEEE Log Number 9034433.
The above formulation is generally referred to as the covariance method [3]. Note that $N$ is the number of data samples available $(N \geq 2 M+1)$. The least squares solution of (2) for obtaining $\boldsymbol{a}_{M}$ results in

$$
R_{M} a_{M}=r_{M}
$$

where $R_{M}=X_{M}^{T} X_{M}$ and $r_{M}=X_{M}^{T} y(k)$. Note that $R_{M}$ is symmetric but not Toeplitz. It, however, has certain shift invariant properties similar to those found in a Toeplitz matrix [2]-14].

A direct solution of (3) requires the inversion of $R_{M}$ which may be computationally prohibitive for large order systems. By resorting to the split matrix iterative methods [5], we have two alternative solutions depending on whether the input signal is nearly white, or an arbitrary colored sequence. For the latter, Ohsmann's [1] method is applicable and is discussed in Section II. A simple method is proposed in Section III for the former.

\section{The Iteration Procedure}

We briefly summarize the matrix iteration procedure as follows [1]: Split the matrix $R_{M}$ into $R_{M}=T+S$ with $E\left\{R_{M}\right\}=E\{T\}$ where $T$ is a Toeplitz approximation of $R_{M}$. One such approximation is given in [1], where the elements of $T, t(i)$ are given by

$$
t(i)=\frac{1}{M+1-i} \sum_{j=0}^{M-i} r_{t}(i+j, j) . \quad i=0,1, \cdots, M
$$

where $r_{x}(i+j, j)$ are the elements of $R_{M}$. We then have

$$
a_{M}^{(n+1)}=a_{M}^{(0)}+a_{M}^{(n)}-T^{-1} R_{M} a_{M}^{(n)}
$$

where $\boldsymbol{a}_{M}^{(0)}=T^{-1} \boldsymbol{r}_{M}$ is the initial condition. The iterative solution of $a_{M}^{(n)}$ converges to the solution $a_{M}$ of (3) provided the spectral radius of $\left(I_{M+1}-T^{-1} R_{M}\right)$ is less than unity, where $I_{M+1}$ is an $M$ $+1 \times M+1$ unity matrix. Since $R_{M}$ is obtained as a product of two nonsquare Toeplitz matrices [see (3)] and $T$ is a Toeplitz approximation of $R_{M}$, the convergence condition is always met with a strong probability.

We now determine an expression for the convergence factor $\rho_{a}^{(n)}$, a measure of the convergence rate of the iteration. From (4), we can obtain the coefficient error vector

$$
\boldsymbol{e}_{a}^{(n)}=-T^{-1} S \boldsymbol{e}_{a}^{(n-1)}=\left(-T^{-1} S\right)^{n} \boldsymbol{e}_{a}^{(0)}
$$

where we have made use of (3) and defined $\boldsymbol{e}_{a}^{(i)}=a_{M}^{(j)}-a_{M}, j=$ $0,1, \cdots, n$. Taking norms on both sides of $(5 a)$ yields the convergence parameter

$$
\rho_{a}^{(n)}=\left(\frac{\left\|\boldsymbol{e}_{a}^{(n)}\right\|_{2}}{\left\|\boldsymbol{e}_{a}^{(0)}\right\|_{2}}\right)^{2} \leq\left(\left\|T^{-1}\right\|\|S\|\right)^{2 n}
$$

where $\|.\|_{2}$ is the $1-2$ norm and $\|$.$\| is the spectral norm$ 
define the following error vectors

$$
\begin{aligned}
& \boldsymbol{e}_{r}^{(m)}=R_{M} a_{M}^{(n)}-r_{M}=R_{M}\left[a_{M}^{(n)}-a_{M}\right]=R_{M} e_{u}^{(n)} \\
& \boldsymbol{e}_{r}^{(0)}=R_{M}\left[a_{M}^{(0)}-a_{M}\right]=R_{M} e_{u}^{(0)} .
\end{aligned}
$$

Note that the vector $R_{M} a_{M}^{(n)}$ is not required to be computed separately. but is available as part of (4) at each iteration. The $l-2$ norm of errors in (6) can be represented as

$$
\left\|e_{r}^{(n)}\right\|_{2} \leq\left\|R_{M}\right\|\left\|e_{a}^{(m)}\right\|_{2} \text { and }\left\|e_{r}^{(0)}\right\|_{2} \leq\left\|R_{M}\right\|\left\|e_{a}^{(0)}\right\|_{2} \text {. }
$$

We can now use the ratio between the two as a measure of convergence and can be related to (5b) yielding

$$
\rho_{r}^{(n)}=\left(\frac{\left\|\boldsymbol{e}_{r}^{(n)}\right\|_{2}}{\left\|\boldsymbol{e}_{r}^{(n)}\right\|_{2}}\right)^{2} \leq\left(\frac{\left\|\boldsymbol{e}_{1 !}^{(n)}\right\|_{2}}{\left\|\boldsymbol{e}_{a}^{(0)}\right\|_{2}}\right)^{2} \leq\left(\left\|T^{-1}\right\|\|S\|\right)^{3 n} .
$$

\section{A. Derivation of Upper Bound on $\rho^{(1)}$}

Consider that the FIR filter input $x(k)$ is white Gaussian with zero mean. As a result the matrix $R_{M}$ is diagonal with entries ( $N$ $-M) \sigma_{i}^{2}$, where $\sigma_{i}^{2}$ is given by (see (2), (3))

$$
\sigma_{i}^{2}=\sum_{j=0}^{N-M-1} x(k-i-j)^{2}, \quad i=0,1, \cdots, M .
$$

We then obtain the spectral norm of $R_{M}$ as follows $(5$, p. 9):

$$
\left\|R_{M}\right\|=\frac{\left\|R_{M} z\right\|_{2}}{\|z\|_{2}} \approx(N-M) \sigma^{2}
$$

where $z$ is an $M+1 \times 1$ vector with unity entries every where yielding the $l-2$ norm $\|z\|_{2}=\sqrt{M+1}$, and $\sigma^{2}$ is the variance of the input process. The spectral norms of $T^{-1}$ and $S$ are then obtained as mean and standard deviation of $\left\|R_{M}\right\|$, given by [6]

$$
\left\|T^{-1}\right\|=\frac{1}{(N-M-1) \sigma^{2}} \text { and }\|S\|_{1}=\sqrt{2(N-M-1)} \sigma^{2} .
$$

From (11) we have the bound

$$
\rho_{r}^{(n)} \leq\left(\frac{2}{N-M-1}\right)^{n}, \quad n \geq 0 .
$$

Even though the upper bound on $\rho_{r}^{(n)}$ has been derived for a white noise input assumption, the validity of this expression for a colored noise situation is demonstrated by the experimental results as detailed in Section IV.

B. Computation of $a_{M}^{(0)}$ and $T^{-1}$

From (3), we now obtain an augmented equation given by

$$
\left[\begin{array}{ll}
r_{y}(0) & r_{M}^{T} \\
r_{M} & R_{M}
\end{array}\right]\left[\begin{array}{c}
1 \\
-a_{M}^{(0)}
\end{array}\right]=\bar{R}\left[\begin{array}{c}
1 \\
-a_{M}
\end{array}\right]=\left[\begin{array}{l}
\epsilon_{y}^{M} \\
0_{M+1}
\end{array}\right]
$$

where $r_{v}(0)=\boldsymbol{y}^{T}(k) \boldsymbol{y}(k)$ and $\epsilon^{M}=r_{*}(0)-\boldsymbol{a}_{M}^{T} \boldsymbol{r}_{M}$, and $O_{M+1}$ is a vector of zeros of order $M+1$. Introducing a vector $\boldsymbol{b}_{M}=[1$ $\left.b_{1}^{M} \cdots b_{M-1}^{M} b_{M}^{M}\right]$ and its reverse vector $\tilde{\boldsymbol{b}}_{M}=\left[b_{M}^{M} b_{M-1}^{M} \cdots b_{1}^{M}\right.$ 1 ] into (13) yields the following recursions, for $m=1,2, \cdots$, $M[2]$ :

$$
\begin{aligned}
& \boldsymbol{b}_{m}=\left(\begin{array}{l}
\boldsymbol{b}_{m-1} \\
0
\end{array}\right)+K_{x}^{m}\left(\begin{array}{l}
0 \\
\tilde{\boldsymbol{b}}_{m-1}
\end{array}\right) \\
& a_{m}=\left(\begin{array}{l}
a_{m-1} \\
0
\end{array}\right)+K_{y}^{m} \tilde{b}_{m} \\
& \text { where } K_{r}^{m}=\Delta_{x}^{m-1} / \epsilon_{x}^{m-1} \text { and } K_{y}^{m}=\Delta_{y}^{m-1} / \epsilon_{x}^{m} \text {, and } \\
& \Delta_{x}^{m-1}=r_{x}(m)+\sum_{\substack{i=1 \\
m-1}}^{m-1} b_{i}^{m-1} r_{\mathrm{v}}(m-i) \\
& \Delta_{y}^{m-1}=r_{x y}(m)-\sum_{i=0}^{m-1} a_{i}^{m-1} r_{x}(m-i) \\
& \epsilon_{i}^{m \prime}=\epsilon_{i}^{m-1}+K_{r}^{m \prime} \Delta_{t}^{m \cdots-1} \text {. }
\end{aligned}
$$

where we have assumed that $0 \leq \alpha<1 / 3$ and $\boldsymbol{M}$ is large, say $M$ $>10$. Comparing (12) and (19), we notice that (19) does not contain the terms $N$, the number of data, and $M$, the FIR model order. However, implicitly, (19) is a function of both $M$ and $N$ : depen-

\footnotetext{
'The correlation coefficient at lag one is defined as $\alpha=r_{1}(1) / r_{x}(0)$, where $r_{1}(0)$ and $r_{1}(1)$ are the autocorrelation function values at lag zero and lag one, respectively. For a single pole process, $\alpha$ indicates the value of the pole.
}

In addition to the recursions in (14), we can also compute $\epsilon_{y}^{\prime \prime}$ recursively which can be used as a recursion stopping parameter if desired. Finally, we need the following initial conditions to start

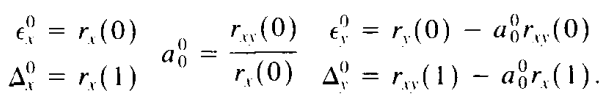

At the end of $M$ recursions in (14), we have $\boldsymbol{a}_{M}^{(0)}$ that goes into (4) and $T^{T}$ is obtained as Cholesky decomposition $[4]: T^{-}=$ and $E=\operatorname{diag}\left(\epsilon_{x}^{0} \epsilon_{x}^{l} \cdots \epsilon_{x}^{M}\right)$ is a diagonal matrix.

\section{A Simplified Iteration Method}

In this method we consider that the input data $x(k)$ is nearly white which results in a banded autocorrelation matrix $R_{M}$. In such of $R_{M}$ with zeros along the principal diagonal. The following iteration procedure is considered

$$
\boldsymbol{a}_{M}^{(n+1)}=\boldsymbol{a}_{M}^{(0)}+\boldsymbol{a}_{M}^{(m)}-D^{-1} R_{M} a_{M}^{(n)}
$$

where $a_{M}^{(0)}=D^{-1} r_{M}$. One immediate advantage of this method is its computational savings. Note that no matrix inversion is required and we only need one matrix vector multiplication per iteration.

The convergence of (16) requires that the largest eigenvalue of the matrix $\left(I_{M+1}-D^{-1} R_{M}\right)$ be less than unity. For example, for a pure white noise input case, the term $D^{-1} R_{M}$ yields a unity matrix and the convergence is assured. However, for a nonwhite or colored input process having a fast decaying autocorrelation function the input process at lag one' $\alpha$, which guarantees the convergence of the iteration (16). The Appendix presents a derivation for this bound, given by $0<\alpha<1 / 3$. Thus, if we know that $0<\alpha<$

\section{Facto} implified algorithm

$\rho_{s}^{(n)}=\left(\frac{\left\|e_{r}^{(n)}\right\|_{2}}{\left\|e_{r}^{(0)}\right\|_{2}}\right)^{2} \leq\left(\frac{\left\|e_{a}^{(\prime \prime)}\right\|_{2}}{\left\|e_{a}^{(0)}\right\|_{2}}\right)^{2} \leq\left(\left\|D^{-1}\right\|\left\|S_{s}\right\|\right)^{2 n}=(\|Q\|)^{2 n}$

where $Q=\left(I_{M+1}-D^{-1} R_{M}\right)$ (see (A2)). We now obtain the bound on $\rho_{s}^{\prime \prime \prime}$ as a function of the spectral radius of the matrix $Q$. Since matrix $Q$ is also symmetric. For a square symmetric matrix, the spectral norm is equal to its spectral radius [5, theorem 1.3]. From

$$
\|Q\|=\lambda_{\max }=\frac{2 \alpha}{1-\alpha} .
$$

Consequently, from (17), (18), the bound on the convergence factor is obtained as

$$
\rho_{s}^{(n)} \leq\left(\frac{2 \alpha}{1-\alpha}\right)^{2 n}, \quad n \geq 0
$$

.

(1)

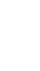
De propose the splitting of $R_{M}$ into $R_{M}=D+S_{s}$, where 0.333 a priori, then we can use the simplified algorithm. . 


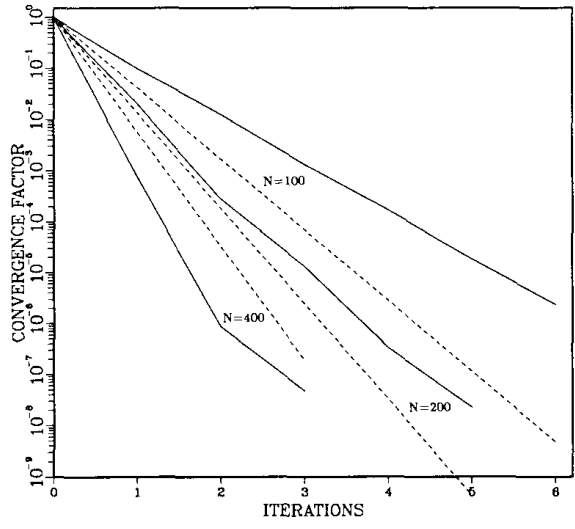

Fig. 1. Convergence performance of method I for colored noise input ( $\alpha$ $=0.8)$ : measured; $\cdots$ theoretical.

dence of (19) on $M$ may be seen in (A3); from the bound that $N \geq$ $2 M+1$, the convergence factor depends on $N$. Also, in obtaining the results in the Appendix, we have assumed that the autocorrelation matrix is both symmetric and Toeplitz. We require a large data to satisfy this assumption. Nevertheless, the convergence factor primarily depends on the value of $\alpha(0 \leq \alpha<1 / 3)$.

\section{Simulation Results}

We considered both white noise and colored noise inputs in evaluating the convergence performance of the two iterative methods discussed here. The colored input data is generated as a single pole process $x(k)=\alpha x(k-1)+w(k)$, where $w(k)$ is a pure white noise process. Also, we present results on the identification of FIR systems in the presence of noise. The results are presented as plots of measured convergence factors versus the number of iterations We shall refer to the iteration method discussed in Section II as method I, and the simplified method of Section III as method II.

The reference (unknown) system considered is a 24th order linear phase FIR filter taken from [8]. Fig. I shows the convergence performance of method I where the input sequence $x(k)$ is generated using $\alpha=0.80$. The dashed lines indicate the bounds set by (12), and the solid lines show the convergence performance of the method for different data lengths, $N=100, N=200$, and $N=$ 400. We have observed that the convergence factor obeyed the bound set by (12) only for $N>300$. This may be attributed to some assumptions that are made in deriving the bound (see Section II).

Fig. 2 shows the convergence performance of method II where the input $x(k)$ is obtained using $\alpha=0.2$. For the same $\alpha$ and $N$ method I always converged faster than method II. The dashed line indicates the bound set by (19) and the solid lines are due to measurement. We have noticed that the convergence has slowed down with increasing $\alpha$, and also required more data points $N$. For example, when $\alpha=0.3$, the iteration did not converge at $N=200$. Also, we have observed divergence of the iteration for $\alpha>1 / 3$.

Let us now consider the FIR system identification in noise where $y(k)$ has been obtained as a sum of the reference (unknown) system output $y^{\prime}(k)$ and an uncorrelated white noise $l^{\prime}(k)$. The signal-tonoise ratio (SNR) at the output is defined as SNR $=10 \log$, $\left(\sigma_{y^{\prime}}^{2} / \sigma^{2}\right)$, where $\sigma^{2}$ is the variance of the reference system output $y^{\prime}(k)$ and $\sigma^{2}$. is the variance of the additive noise $v^{\prime}(k)$.

The convergence performance of method $I$ for the additive noise case is shown in Fig. 3. The solid lines indicate the no noise case and the two broken lines the additive noise case. Notice that the convergence rate of the iteration has not been affected much by the additive noise at the output. Fig. 4 shows the performance of method II in noise. We observe that the convergence rate is highly affected by the noise at low data lengths (note the $N=100$ case)

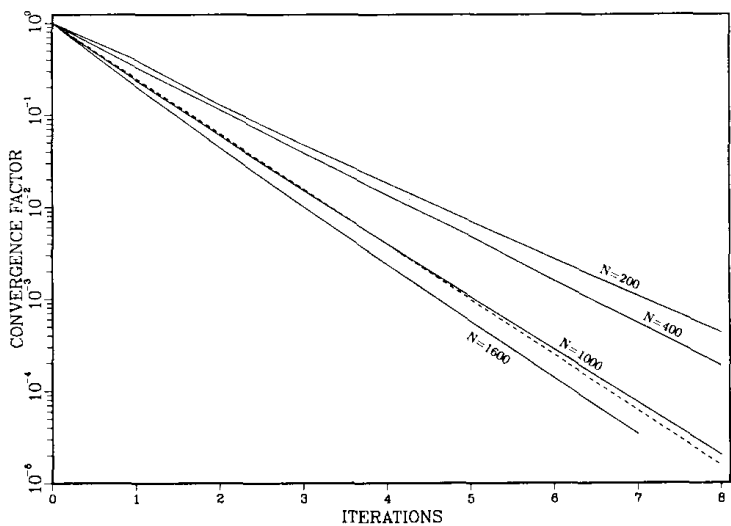

Fig. 2. Convergence performance of method II for colored noise input ( $\alpha$ $=0.2)$ : - measured: - - theoretical.

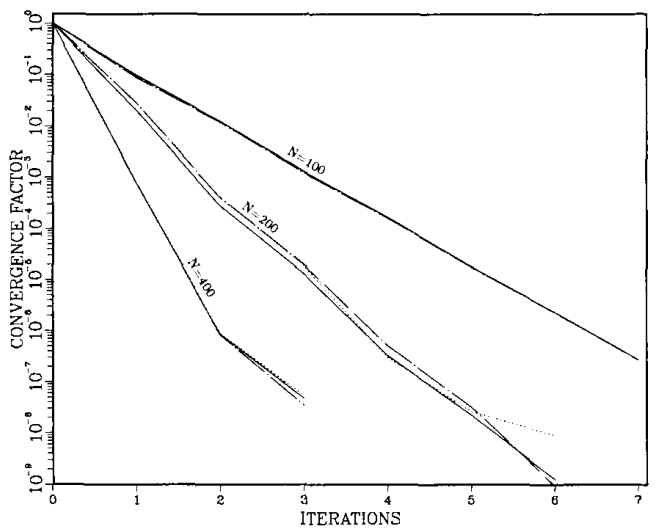

Fig. 3. Convergence performance of method I in noise with white noise input: $\ldots$ SNR $=\infty: \cdots \cdot$ SNR $=10 \mathrm{~dB}:-\cdot-\cdot$ SNR $=$ $0 \mathrm{~dB}$.

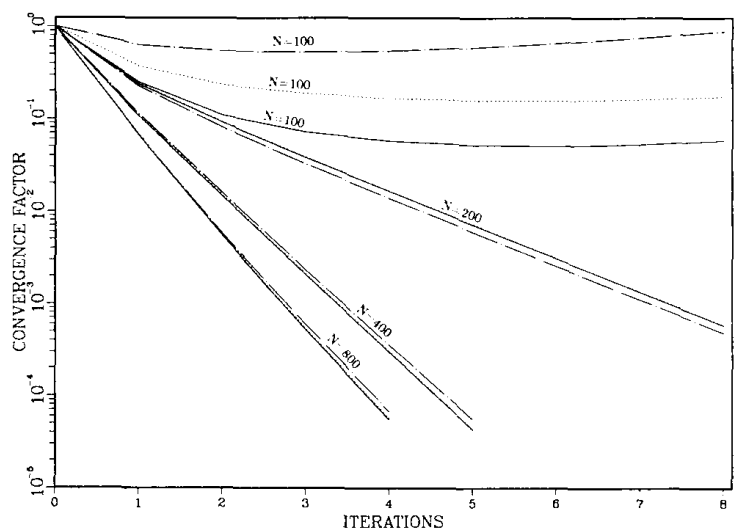

Fig. 4. Convergence performance of method II in noise with white nois input: $\ldots \mathrm{SNR}=\infty ; \cdots \cdot \mathrm{SNR}=10 \mathrm{~dB} \cdot \ldots \cdot-\mathrm{SNR}=$ $0 \mathrm{~dB}$

However. for $N \geq 200$, the convergence rate with noise added at the output is comparable to that of the no noise case.

V. Conclusions

We presented two iterative methods based on matrix splitting to solve the least squares identification of FIR systems. The first 
method extends a recently proposed procedure by Ohsmann [1] to an order recursive solution. This method does not involve any direct matrix inversion and solves the initial estimate by incorporating a lattice algorithm. We also derived a more accurate expression for the convergence factor which indicates the convergence performance of the iteration (4). The second method is a simplified iterative procedure for situations where the input is known to be white or nonwhite with a correlation coefficient of value $\alpha<1 / 3$ This method does not require any matrix inversion and is computationally efficient. Simulation results indicate satisfactory performance of both methods. Results have also been presented for identification in the presence of noise.

\section{APPENDIX}

From (16) we have the matrix $Q=\left(I_{M+1}-D^{-1} R_{M}\right)$. Let us consider that the input $x(k)$ has been obtained as a single pole process $x(k)=\alpha x(k-1)+w(k)$, where $w(k)$ is a zero mean white Gaussian process with unity variance and $\alpha$ is the correlation coefficient. In this derivation, we assume that $0 \leq \alpha<1$ without any loss of generality. We shall also consider that the resulting autocorrelation matrix $R_{M}$ is symmetric and Toeplitz. Therefore $Q$ has zero entries along the principal diagonal. The characteristic equation $\Delta_{m}=\left|Q-\lambda I_{M+1}\right|=0$, can be written in the following recursive form [7]:

$$
\begin{aligned}
\Delta_{m}(\lambda)= & -\left[2 \alpha^{2}-\lambda\left(1+\alpha^{2}\right)\right] \Delta_{m-1}(\lambda) \\
& -\alpha^{2}(1+\lambda)^{2} \Delta_{m-2}(\lambda), \quad m=1,2, \cdots, M
\end{aligned}
$$

where $\Delta_{-1}(\lambda)=1$, by definition, and $\Delta_{0}(\lambda)=-\lambda$. After a few manipulations we can rewrite (A1) as

$$
\Delta_{m}(\lambda)=-[\alpha(1+\lambda)]^{m}\left[\frac{\lambda \sin (m+1) x}{\sin x}+\frac{\alpha(1+\lambda) \sin m x}{\sin x}\right] .
$$

Equation (A2) then yields $M+1$ distinct eigenvalues given by [7]

$$
\begin{gathered}
\lambda_{m}^{M}=-2 \frac{\alpha^{2}-\alpha \cos \left(\frac{(m+1) \pi}{M+2}\right)}{1-2 \alpha \cos \left(\frac{(m+1) \pi}{M+2}\right)+\alpha^{2}} \\
m=0,1,2, \cdots, M .
\end{gathered}
$$

The maximum eigenvalue corresponds to the case $m=0$ in (A3) and is given by

$$
\lambda_{\max }=-2 \frac{\alpha^{2}-\alpha \cos \left(\frac{\pi}{M+2}\right)}{1-2 \alpha \cos \left(\frac{\pi}{M+2}\right)+\alpha^{2}} \approx \frac{2 \alpha}{1-\alpha}
$$

where we let $M$ be large such that $\cos (\pi / M+2) \approx 1$ in (A4). The convergence condition requires that $\left|\lambda_{\max }\right|<1$ which yields the bound $\alpha<1 / 3$.

\section{REFERENCES}

[1] M. Ohsmann, "An iterative method for the identification of MA systems," IEEE Trans. Acoust., Speech, Signal Processing, vol. ASSP36, pp. 106-109, no. 1, Jan. 1988.

[2] S. L. Marple, "Efficient least squares FIR system identification," JEEE Trans. Acoust., Speech, Signal Processing, vol. ASSP-29, no. 1, pp. 62-73, Feb. 1981.
[3] S. Haykin, Adaptive Filter Theory. Englewood Cliffs, NJ: PrenticeHall, 1986

[4] B. Friedlander, "Lattice filters for adaptive filtering," Proc. IEEE, vol. 70 , no. 8, pp. 829-867, Aug. 1982

15] R. S. Varga, Matrix Iterative Analysis. Englewood Cliffs, NJ: Prentice-Hall, 1962.

[6] H. Cramér, Mathematical Methods of Statistics. Princeton, NJ: Princeton University Press, 1946. pp. 341-363.

17] U. Grenander and G. Szegö. Teoplitz Forms and Their Applications. Berkeley, CA: University of California Press, 1958, pp. 62-80.

[8] L. R. Rabiner, R. E. Chrochiere, and J. B. Allen, "FIR system identification in the presence of noise and with band-limited inputs," IEEE Trans. Acoust., Speech, Signal Processing, vol. ASSP-26, no. 4, pp. 319-333, Aug. 1978.

\section{Weighted Averaging of a Set of Noisy Images for Maximum Signal-to-Noise Ratio}

MICHAEL UNSER AND MURRAY EDEN

\begin{abstract}
This paper considers the problem of estimating a signal from a weighted average of $N$ registered noisy observations. A set of optimal weighting coefficients is determined by maximizing a signal-tonoise ratio criterion. This solution can be computed by first standardizing each observation with respect to its first and second moments and then evaluating the first eigenvector of the corresponding $N \times N$ innerproduct matrix. The resulting average is shown to be proportional to the first basis vector of the Karhunen-Loève transform provided that the data has been standardized in an appropriate fashion. The low sensitivity of this approach to the presence of outliers is illustrated by using real electron micrographs of ostensibly identical virus particles.
\end{abstract}

\section{INTRODUCTION}

Given a series of noisy observations of an unknown signal, the simplest and often most efficient noise reduction technique is averaging [1, p. 434]. An interesting application of this technique is correlation averaging, which is now used routinely for improving signal-to-noise ratios of electron micrographs of quasi-periodic arrays, or sets of images of ostensibly identical free standing particles [2]-[4]. In this approach, single unit cells or individual particle images are brought into translational and rotational alignment [3]-[5], analyzed using multivariate statistical methods to identify subsets of similar particles and eliminate outliers [6], [7], and finally averaged. The quality of the restored signal is typically assessed in terms of estimated signal-to-noise ratios [4], [8].

An important step in this whole procedure-although not usually emphasized-is the preprocessing where the individual observations are normalized to compensate for varying acquisition parameters. This is usually achieved by simple linear rescaling of the intensity distributions. In most applications, the weighting coefficients are chosen to standardize the range ( $\mathrm{min} / \mathrm{max}$ normalization) or the first and second moments (moment normalization) of the intensity distribution of each observation [9].

In effect, during this analysis the signal is estimated from a weighted average of the initial registered noisy observations. Obviously, there are many ways of selecting the weighting coefficients

Manuscript received October 4, 1988; revised August 2, 1989.

M. Unser is with the Biomedical Engineering and Instrumentation Branch, the National Institutes of Health. Bethesda, MD 20892, and with INSERM, Unit 138, F-94010 Créteil Cedex. France.

M. Eden is with the Biomedical Engineering and Instrumentation Branch, the National Institutes of Health. Bethesda. MD 20892.

IEEE Log Number 9034435. 